\title{
Tuberculosis of the Cervix Mimicking Carcinoma
}

\author{
Angeline Hui Kiang Chua ${ }^{a}$,, Timothy Yong Kuei Lim ${ }^{\mathrm{b}}$
}

\begin{abstract}
This is a rare case of a 35-year-old lady who presented initially to the reproductive clinic for failure to conceive after 5 years and also complained of persistent post-coital bleeding for the past 1 year. A $3-\mathrm{cm}$ exophytic cervical lesion was noted on colposcopy and the impression then was of cervical cancer. Computed tomography (CT) scan of the abdomen showed multiple peritoneal nodules and magnetic resonance imaging (MRI) pelvis showed a 3.4-cm cervical tumor with possible parametrial invasion. Both results were also suggestive of malignancy. Histology, however, returned as caseating granulomatous inflammation compatible with tuberculosis. There was complete resolution of the cervical lesion after 3 months of anti-tuberculosis treatment. Tuberculosis of the cervix is an extremely rare initial presentation of tuberculosis. Hence, there should be a high index of suspicion during colposcopy for tuberculosis when low-risk patients are found to have a cervical lesion.
\end{abstract}

Keywords: Tuberculosis; Cervix; Carcinoma

\section{Introduction}

Tuberculosis of the cervix is a rare initial presentation of tuberculosis, especially in developed countries like Singapore. It accounts for $0.1-0.65 \%$ of all cases of tuberculosis, and $5-24 \%$ of genital tract tuberculosis [1].

Genital tuberculosis is usually secondary to tuberculosis elsewhere in the body, usually pulmonary. It usually spreads by hematogenous, lymphatic or direct extension with an intraabdominal or peritoneal focus [2]. It is also more common in patients who are immunocompromised, such as those who are positive for the human immunodeficiency virus.

Patients usually present with persistent vaginal discharge, abnormal bleeding and other constitutional symptoms of infection such as weight loss, fatigue and fever. It can also be a

Manuscript submitted April 30, 2018, accepted May 2, 2018

aDepartment of Obstetrics and Gynecology, KK Women's and Children's Hospital, Singapore

'Department of Gynecological Oncology, KK Women's and Children's Hospital, Singapore

${ }^{\mathrm{c}}$ Corresponding Author: Angeline Hui Kiang Chua, 256 Compassvale Rd, \#14-684, Singapore 540256. Email: angeline.chua2@mohh.com.sg

doi: https://doi.org/10.14740/jmc3074w cause of infertility in young patients.

We report a case of cervical tuberculosis, which was initially highly suspicious of a malignant lesion on clinical examination.

\section{Case Report}

A 36-year-old nulliparous Filipino lady residing in Singapore presented initially to the subfertility clinic for failure to conceive after 5 years. She also complained of persistent post-coital bleeding for 1-year duration. There was no fever, night sweats, cough or other constitutional symptoms. She had no positive contact history with any patients who had tuberculosis. She also had no significant past medical history and is a non-smoker.

On examination, there was a $3-\mathrm{cm}$ exophytic, friable lesion with contact bleeding noted on the cervix. The patient was referred to the colposcopy clinic urgently for further evaluation. The impression by an experienced colposcopist was of cervical malignancy (Figs. 1 and 2).

However, Pap smear was negative for malignant cells. Interestingly, the cervical punch biopsy revealed multiple caseating granulomatous inflammation compatible with tuberculosis (Fig. 3). The Ziehl-Neelsen stain showed acid fast bacilli, compatible with Mycobacterium tuberculosis.

A staging work-up was ordered initially in view of the high suspicion for malignancy. Chest X-ray showed scarring with bronchiectatic changes in both lung apices. There were no obvious pulmonary lesions seen. Magnetic resonance imaging (MRI) pelvis showed a cervical tumor with possible parametrial invasion, measuring up to $3.4 \mathrm{~cm}$ in maximal dimension

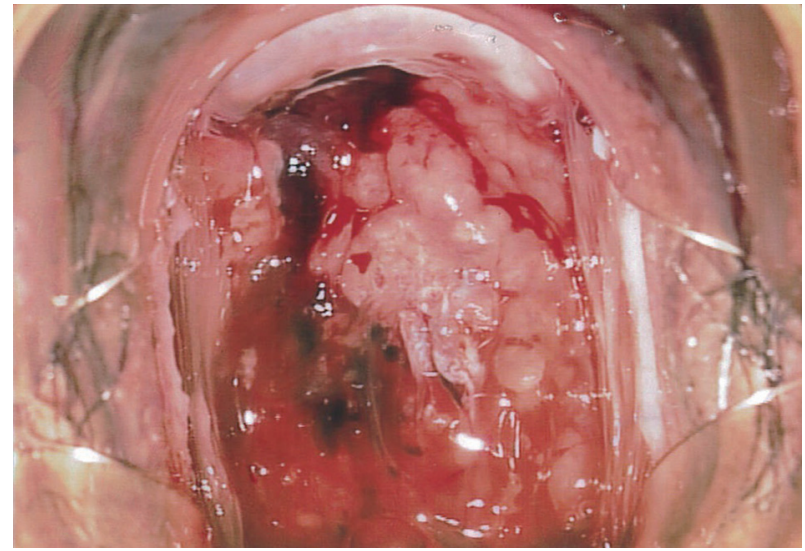

Figure 1. A 3-cm exophytic lesion with contact bleeding noted on colposcopy examination, highly suspicious of malignancy. 


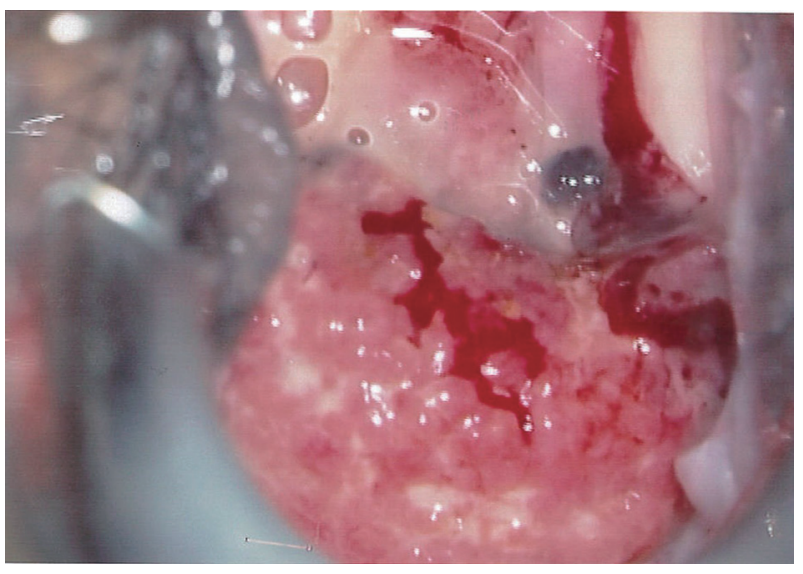

Figure 2. Friable lesion with contact bleeding seen again on colposcopy 5 days after initial biopsy, before starting anti-tuberculosis treatment.

\section{(Figs. 4 and 5).}

Computed tomography (CT) abdomen reported innumerable enhancing peritoneal nodules in the mesentery, omentum, liver surface and serosal surface of the bowel, largest $0.7 \mathrm{~cm}$. This was highly suggestive of peritoneal carcinomatosis when the initial impression was of cervical malignancy. There was no pelvic lymphadenopathy.

The patient was referred to the infectious disease physician immediately when the biopsy report returned. Her sputum, laryngeal swabs and urine cultures were all negative for tuberculosis. She was started on rifampicin, isoniazid, pyrazinamide and ethambutol.

There was marked reduction in the cervical mass size 2 months after starting anti-tuberculosis medications, with complete resolution of the cervical lesion 3 months later (Fig. 6). Patient completed 9 months of treatment in total.

\section{Discussion}

Genital tuberculosis most commonly affect the fallopian tube

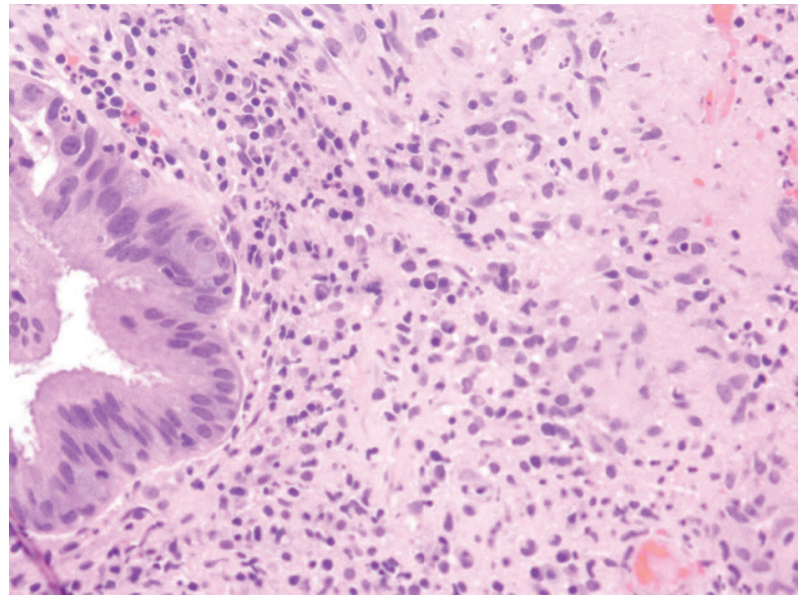

Figure 3. Cervical mucosa with caseating granuloma on cervical biopsy specimen.

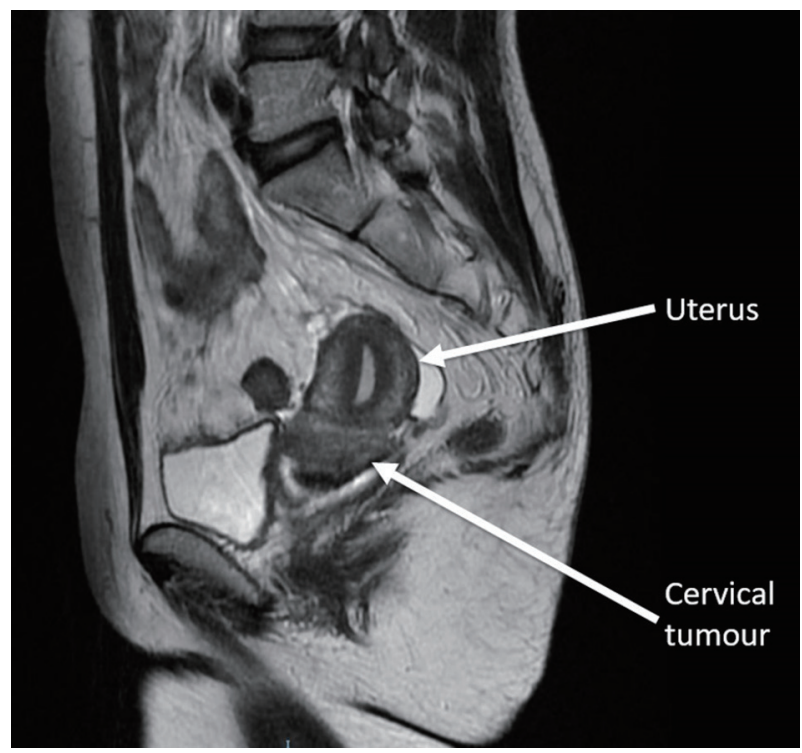

Figure 4. Magnetic resonance imaging of the pelvis T2-weighted sagittal view showed the cervical tumour.

(95-100\%), endometrium (50-60\%) and ovaries (20-30\%) [3]. Pelvic organs are infected from a primary focus, most commonly a pulmonary source, by hematogenous spread. The cervix is infected by lymphatic spread or by direct extension. Cervical tuberculosis is rarely a primary infection.

In our patient, the chest X-ray which showed bronchiectatic changes is likely to be consistent with a previous pulmonary tuberculosis infection. While the focus in the lung usually heals in most cases, the lesion may remain dormant in the genital tract for many years, and can be reactivated later.

Cervical tuberculosis can present as papillary or vegetative lesions, ulcers and miliary growths, therefore mimicking malignancy [3]. The diagnosis is usually made by histology of a cervical biopsy specimen. The gold standard for diagnosis is by culture growth of Mycobacterium tuberculosis. However, one-third of cases can be culture negative [4,5]. Acid fast bacilli are not found to be useful in making the diagnosis, but

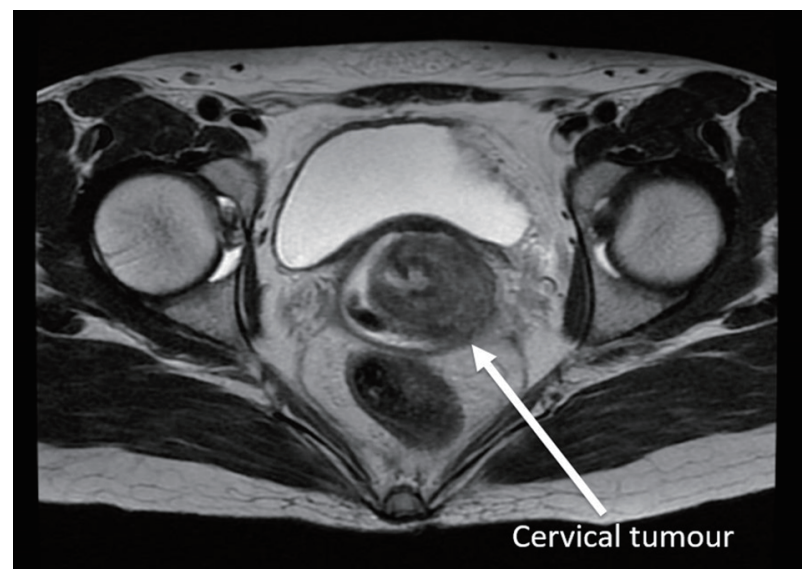

Figure 5. Magnetic resonance imaging of the pelvis T2-weighted axial view showed cervical tumour with parametrial involvement. 


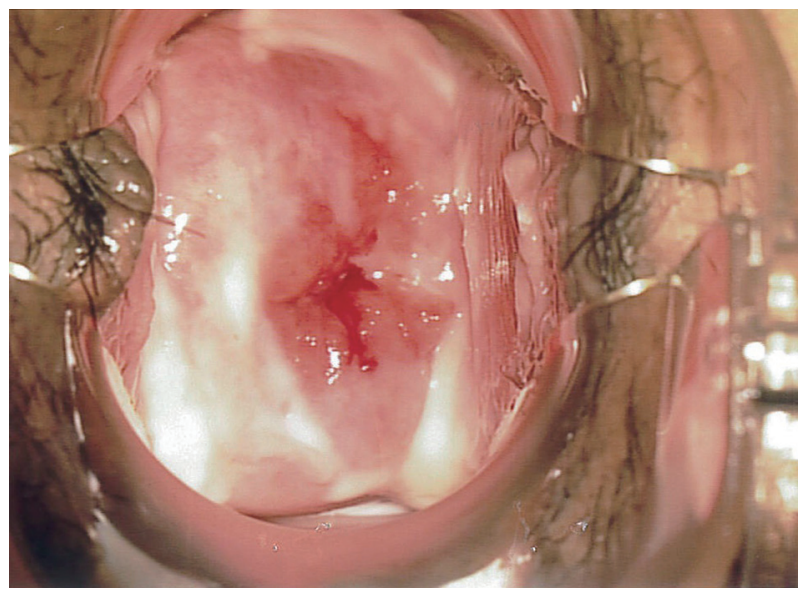

Figure 6. Complete resolution of the cervical lesion 3 months after starting treatment.

the presence of typical granuloma is sufficient for diagnosis if other causes of granulomatous cervicitis are excluded, or a primary focus identified [6].

The treatment regime is similar to that of pulmonary tuberculosis. A cervical lesion should respond to 6 months of standard treatment [6] and is a good marker to assess response to treatment.

Subfertility, the initial presentation in our patient, is common even after treatment, owing to involvement of the fallopian tube and endometrium, as well as subsequent healing by fibrosis [7]. This patient had a hysterosalpingography done as part of the subfertility work-up 6 months after completion of treatment. Both fallopian tubes showed only partial opacification without free intraperitoneal spillage despite repeated attempts, suggestive of obstruction.

Tuberculosis is uncommon in a developed country such as Singapore. The age-standardized incidence rate of tuberculosis in Singapore was 38.7 per 100,000 population in 2016 . Of the 3,100 new cases that were notified in $2016,1,483(47.8 \%)$ of them were non-residents [8]. In Philippines, where our patient resided in before coming to Singapore, tuberculosis is a major health problem. It is the sixth leading cause of death and ill- ness.

\section{Conclusions}

In our modern society with a constant influx of immigrants, there should be a high index of suspicion of tuberculosis in patients with abnormal cervical lesion as it may mimic malignancy.

\section{Conflict of Interest}

Authors declare no conflict of interest.

\section{References}

1. Carter JR. Unusual presentations of genital tract tuberculosis. Int J Gynaecol Obstet. 1990;33(2):171-176.

2. Schaefer G. Female genital tuberculosis. Clin Obstet Gynecol. 1976;19(1):223-239.

3. Chowdhury NN. Overview of tuberculosis of the female genital tract. J Indian Med Assoc. 1996;94(9):345-346, 361.

4. Shobin D, Sall Pellman C. Genitourinary tuberculosis simulating cervical carcinoma. J Reprod Med. 1976;17(5):305-308.

5. Sutherland AM, Glen ES, MacFarlane JR. Transmission of genito-urinary tuberculosis. Health Bull (Edinb). 1982;40(2):87-91.

6. Lamba H, Byrne M, Goldin R, Jenkins C. Tuberculosis of the cervix: case presentation and a review of the literature. Sex Transm Infect. 2002;78(1):62-63.

7. Parikh FR, Nadkarni SG, Kamat SA, Naik N, Soonawala SB, Parikh RM. Genital tuberculosis - a major pelvic factor causing infertility in Indian women. Fertil Steril. 1997;67(3):497-500.

8. Ministry of Health Singapore: communicable diseases surveillance in Singapore 2016. Retrieved from URL: https://www.moh.gov.sg/content/dam/moh_web/Publications/Reports/2017/Other\%20Diseases.pdf. 